Objective To explore preventive effects of intracoronary administration of Anisodamine on myocardial microcirculation perfusion after primary PCI in STEMI patients.

Methods From March 2009 to August 2010, a total of 86 consecutive patients with first STEMI who underwent primary PCI within $12 \mathrm{~h}$ of symptom onset were enrolled in this study. Patients were divided into anisodamine group $(n=43)$ and saline control group $(n=43)$ randomly. The lesion of IRA was treated by percutaneous transluminal coronary angioplasty with over the wire balloon. In anisodamine group, $2000 \mathrm{ug}$ anisodamine (concentration $500 \mathrm{ug} / \mathrm{ml}$ ) was injected into the coronary artery as a preventive measure via over-the-wireballoon. In control group, equal volume of saline was injected into the coronary artery in the same way. After that the lesion was predilated with the balloon. Angiography was done after 5 min of stent implanted, CTFC and TMPG were recorded and compared. 200 ug nitroglycerin was bolus injected intracoronary before angiography to exclude spasm of coronary artery. The major adverse cardiac events (MACE) in hospital of the two groups were compared.

Results There was no significant difference between the two groups in baseline characteristics (all p $>0.05$ ). There were no significant differences in IRA distribution, multi-vessel lesion and the use of tirofiban between the two groups. No significant differences were found in the oral drugs used after pPCI in the two groups (all p $>0.05$ ). Compared to control group, the proportion of patient with TMPG 3 grade in anisodamine group was much higher $(83.72 \%$ vs $62.79 \%$, $\mathrm{p}<0.05)$. The CTFC of each coronary artery in anisodamine group is lower than those in control group (LAD: $21.67 \pm 7.32$ vs $26.92 \pm 8.67$; RCA: $21.05 \pm 6.17$ vs $24.26 \pm 8.51$; LCX: $19.75 \pm 7.13$ vs $23.59 \pm 9.31$, all $p<0.05)$. Comparison of whole frame counts within anisodamine group and control group: $0-13$ counts were 10 cases $(23.26 \%)$ vs five cases $(11.63 \%)$, $13-23$ counts were 29 cases $(67.44 \%)$ vs 22 cases $(51.16 \%)$, $23-40$ counts were four cases $(9.30 \%)$ vs 16 cases $(37.21 \%)$, flow velocity of anisodamine group was obviously quicker than that of control group $(\mathrm{p}<0.05)$. After $5 \mathrm{~min}$ of intracoronary administration of anisodamine, systolic, diastolic and mean coronary pressure found no significant change $(p>0.05)$. The heart rate increased significantly, but no reperfusion arrhythmia happened in anisodamine group, while 10 cases of reperfusion arrhythmia (23.26\%) happened in the control group $(\mathrm{p}<0.05)$.

Conclusion Preventive intracoronary administration of anisodamine can effectively improve myocardial microcirculation perfusion after primary PCI in acute STEMI, and decrease the incidence of reperfusion arrhythmia and MACE in hospital.

\title{
Interventional cardiology
}

[gw22-e0129] PREVENTIVE EFFECTS OF INTRACORONARY ADMINISTRATION OF ANISODAMINE ON MYOCARDIAL MICROCIRCULATION PERFUSION AFTER PRIMARY PCI IN STEMI PATIENTS

Fu Xianghua, Fan Yanming, Wang Xuechao, Wang Yanbo, Gu Xinshun, Fan Weise, Jiang Yunfa, Hao Guozhen The Second Hospital of Hebei Medical University, Hebei, China

10.1136/heartjnl-2011-300867.433 